\title{
Destruction of a Thin-Walled Spherical Vessel by Internal Pressure
}

\author{
V. V. Struzhanov ${ }^{1, \text { a) }}$ and A. E. Chaikin ${ }^{2, b)}$ \\ ${ }^{1}$ Institute of Engineering Science, Ural Branch of the Russian Academy of Sciences, 34 Komsomolskaya St., 620049, \\ Ekaterinburg, Russian Federation \\ ${ }^{2}$ Institute of Natural Sciences and Mathematics, Ural Federal University, 48 Kuibysheva St., 620000, Ekaterinburg, \\ Russian Federation \\ ${ }^{a)}$ Corresponding author: stru@imach.uran.ru \\ b)chaykin.ae@yandex.ru
}

\begin{abstract}
The problem of destruction of a thin-walled spherical vessel by increasing internal pressure is formulated. The material properties are described both at the hardening stage and at the softening stage (prefracture stage). Based on the mathematical theory of catastrophes, the equations of equilibrium are written, and the critical value of pressure is determined when the vessel is destroyed.
\end{abstract}

\section{INTRODUCTION}

Spherical thin-walled vessels are widely used in industry, for example, for storage and transportation of liquefied gas. Moreover, they are influenced by high internal pressure. Their destruction may lead to disastrous consequences. While designing such tanks, it is necessary to calculate as accurately as possible the destruction and determination of the critical internal pressure. The accuracy of the calculation is determined by inclusion of the last, supercritical stage of material deformation (the stage of softening) into consideration.

The maximum possible accuracy of the calculation will be achieved if we consider failure as the inability to maintain the balance of the structural element under a given load. The loss of equilibrium occurs when the material in a certain set of individual areas of the structural element passes to the supercritical stage of deformation (the stage of softening), at which the strength of the material is unstable.

Softening has been repeatedly recorded in experiments [1-4]. However, accounting for such material States has yet to find wide application in calculations for the strength of structural elements due to the complexity of building a material model that includes a description of the behavior of the material at the softening stage and the complexity of the experimental determination of the parameters included in the model. In addition, when such models are used, there are mathematical difficulties associated with non-fulfillment of the Hadamard conditions [5]; that is, the resulting systems of equilibrium equations have non-unique and unstable solutions. Only some problems have been solved (for example, [6-8]).

In this paper, a material model is proposed that takes into account all stages of deformation (including softening) in the plane stress state of the material elements. It is shown how to obtain the necessary parameters for model identification from a simple experiment on torsion of a cylindrical sample with fixing the softening stage. Then, a method for determining the maximum pressure in thick-walled spherical vessels is given. The use of the proposed material model has allowed us to apply the methods of the mathematical theory of catastrophes to solving the formulated problem. 


\section{PROBLEM FORMULATION}

Consider a thin-walled spherical shell with an inner radius $b$. The shell thickness is $t<<$ b. A quasistatically increasing internal pressure $\mathrm{p}$ acts on the shell. The temperature is constant. It is required to find the value of the limiting pressure $\mathrm{p}^{*}$, when the shell is destroyed.

With increasing pressure to failure, the shell material goes through all stages of deformation, namely elasticity, hardening, and softening. We determine the properties of the shell material at all stages of deformation, including the stage of softening. With proportional loading, the theory of small elastic-plastic strains is generally used to calculate the stress-strain state. However, its first postulate of the constancy of the bulk modulus at the softening stage is not fulfilled due to the presence of bulk material failure. Instead of the second postulate of the proportionality of the stress and strain tensor deviators, we will preserve the proportionality of the full stress and strain tensors with some variable matrix coefficient depending on strains. This means that, in the inelastic region, the level lines of the potential energy function are similar to the level lines in the elastic region [9]. In this case, the stress potential is a convex-concave function. In the area of convexity, the material is stable, whereas it is at the stage of softening (unstable) in the area of concavity [9].

Based on the arguments given above, we obtain the following defining relations for the plane-stress state of shell elements [9]:

$$
\left(\begin{array}{l}
\sigma_{1} \\
\sigma_{2}
\end{array}\right)=\psi\left(\varepsilon_{1}, \varepsilon_{2}\right) \cdot \frac{E}{1-\mu^{2}} \cdot\left(\begin{array}{ll}
1 & \mu \\
\mu & 1
\end{array}\right) \cdot\left(\begin{array}{l}
\varepsilon_{1} \\
\varepsilon_{2}
\end{array}\right),
$$

where $\mathrm{E}$ is the Young modulus in elasticity, $\mu$ is Poisson's ratio, $\sigma_{1}, \sigma_{2}$ and $\varepsilon_{1}, \varepsilon_{2}$ are, respectively, stresses and strains in a rectangular Cartesian system in a plane element under the plane-stress state. The parameter $\psi$ needs to be defined in the experiment.

Let us use a complete torsion diagram of a cylindrical sample, from which we can obtain a diagram with a descending branch at the stage of surface layer softening under pure shear [10]. Let this diagram be approximated by the expression $\tau=G \cdot\left(\gamma-50 \gamma^{2}\right)$. Here, $\tau$ is the tangent stress, $\gamma$ is shear, and $G=\frac{H}{2 \cdot(1+\mu)}$ is the shear modulus in elasticity.

Then, the strain energy is

$$
V=\int_{0}^{\gamma} \tau d \gamma=V^{e} \cdot\left(1-\frac{100}{3} \cdot \sqrt{\frac{2 \cdot V^{3}}{G}}\right),
$$

where $V^{e}=\frac{1}{2} \cdot G \gamma^{2}$ is elastic energy, $\gamma=\sqrt{\frac{2 \cdot V^{e}}{G}}$.

In the General case of the plane-stressed state,

$$
V^{e}\left(\varepsilon_{1}, \varepsilon_{2}\right)=E\left[2 \cdot\left(1-\mu^{2}\right)\right]^{-1} \cdot\left(\varepsilon_{1}^{2}+2 \mu \varepsilon_{1} \varepsilon_{2}+\varepsilon_{2}^{2}\right)
$$

Since $\sigma_{1}=\frac{d V}{d \varepsilon_{1}}, \sigma_{2}=\frac{d V}{d \varepsilon_{2}}$, we find from equality (1) that

$$
\psi=1-100 \cdot \sqrt{V^{e} \cdot \frac{1+\mu}{E}} .
$$


Only strains occur (plane-stress state) in the spherical shell, $\varepsilon_{1}=\varepsilon_{2}=\varepsilon_{\theta}=\varepsilon_{\varphi}$; then, from formula (2), we find that $\psi=1-100 \varepsilon \cdot \sqrt{\frac{1+\mu}{1-\mu}}$.

Hence, $\sigma_{\vartheta}=\sigma_{\varphi}=\sigma=\left(1-100 \varepsilon \cdot \sqrt{\frac{1+\mu}{1-\mu}}\right) \frac{E}{1-\mu} \varepsilon$.

Figure 1 shows a qualitative view of the stress potential obtained above for the plane-stress state. Convexity (hardening) and concavity (softening) areas are clearly visible.

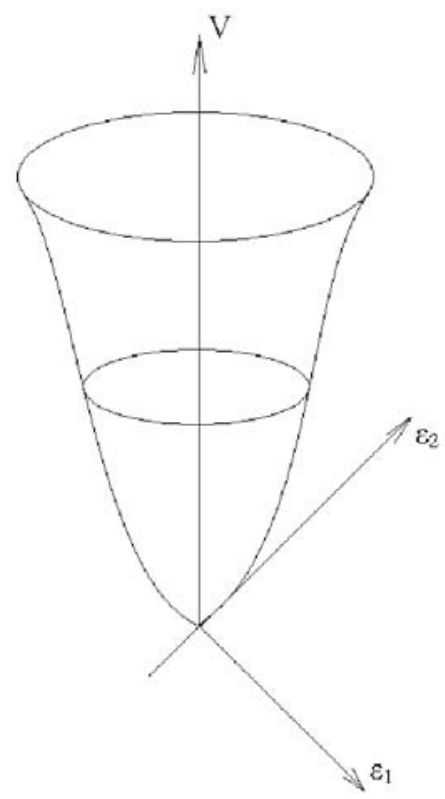

FIGURE 1. Qualitative view of the stress potential

\section{EQUILIBRIUM EQUATION}

We write the Lagrangian of the system, which, under quasistatic loading, is represented as the sum of the potential energy of the deformation of a spherical shell and the work of external forces, taken with a minus sign. The energy of a sphere element is

$$
\frac{1}{2} \cdot\left(\sigma_{\theta} \varepsilon_{\theta}+\sigma_{\varphi} \varepsilon_{\varphi}\right)=\left(1-100 \varepsilon \sqrt{\frac{1+\mu}{1-\mu}}\right) \cdot \frac{E \varepsilon}{1-\mu} \varepsilon=\left(1-100 \frac{v}{b} \cdot \sqrt{\frac{1+\mu}{1-\mu}}\right) \cdot \frac{E}{1-\mu} \frac{v^{2}}{b^{2}} .
$$

Here, $v$ is the radial displacement of points on the inner surface of the sphere, $\varepsilon=v / b$. Then, the total energy (Lagrangian) of the system is the function

$$
W=4 \pi b^{2} t \cdot\left(1-100 \frac{v}{b} \cdot \sqrt{\frac{1+\mu}{1-\mu}}\right) \cdot \frac{E}{1-\mu} \frac{v^{2}}{b^{2}}-4 \pi b^{2} p v
$$


where $4 \pi \mathrm{b}^{2} \mathrm{t}$ is the volume of the sphere, $4 \pi \mathrm{b}^{2}$ is the area of the inner surface. Note that the displacement $v$ is a parameter of the state of the system and that the internal pressure $p$ is the system control parameter. Now, the equilibrium equation has the form [11]

$$
\frac{d W}{d v}=\frac{2 E t v}{b^{2}(1-\mu)}\left(1-150 \frac{v}{b} \cdot \sqrt{\frac{1+\mu}{1-\mu}}\right)-p b^{2} .
$$

From equation (3), we obtain the relationship between the control parameter and the state parameter, namely

$$
p=\frac{2 E t v}{b^{2}(1-\mu)}\left(1-150 \frac{v}{b} \sqrt{\frac{1+\mu}{1-\mu}}\right) .
$$

Let us define the maximum of the function $\mathrm{p}(\mathrm{v})$ when the shell will collapse. We have the equation

$$
\frac{d p}{d v}=\frac{2 E t}{b^{2}(1-\mu)}\left(1-300 \frac{v}{b} \sqrt{\frac{1+\mu}{1-\mu}}\right)=0 .
$$

From here, we find the coordinates of the extreme point

$$
v=\frac{b}{300} \sqrt{\frac{1+\mu}{1-\mu}}, p=\frac{E t}{300 b} \sqrt{\frac{1}{1-\mu^{2}}} .
$$

Figure 2 shows the curve of the equilibrium states of the shell up to destruction.

For the numerical values $E=2 \cdot 10^{4} \frac{\mathrm{kg}}{\mathrm{mm}^{2}}, b=10 \mathrm{~mm}, t=1 \mathrm{~mm}, \mu=0.3$. The maximum displacement of the inner surface of the shell $v^{*}=0.045 \mathrm{~mm}$; the maximum (breaking) pressure $p^{*}=7 \mathrm{~kg} / \mathrm{mm}^{2}$.

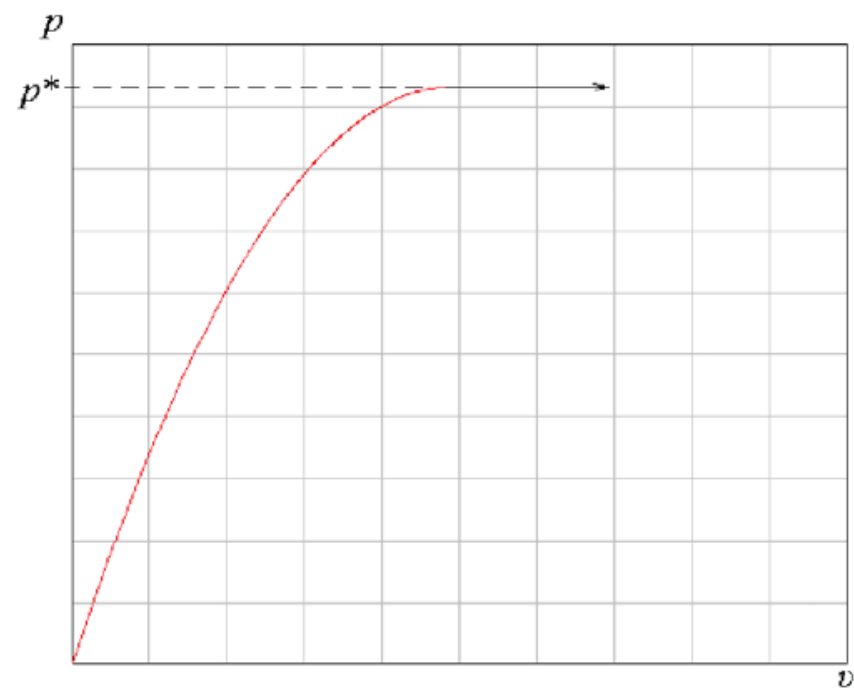

FIGURE 2. The curve of the equilibrium states of the system up to destruction 


\section{CONCLUSION}

A technique for determining the maximum destructive pressure in a thin-walled spherical vessel has been proposed. It is based on a description of the properties of a material with a convex-concave potential taking into account the final stage of deformation (softening), with the subsequent use of the apparatus of the theory of catastrophes.

\section{REFERENCES}

1. V. E. Vildeman and N.G. Chausov, Industrial Laboratory. Diagnostics of Materials 73(10), 55-59 (2007).

2. A. V. Ipatova and V. E. Vildeman, J. Samara State Tech. Univ., Ser. Phys. Math. Sci. 4(29), 106-114 (2012).

3. V. E. Vildeman and M. P. Tretyakov, J. Mach. Manuf. Reliab. 2, 93-98 (2013).

4. V. I. Mironov, Industrial Laboratory. Diagnostics of Materials 68(10), 47-52 (2002).

5. V. Yu. Arsenin, Methods of Mathematical Physics and Special Functions, 2nd Ed. (Nauka, Moscow, 1984).

6. V. V. Struzhanov and V. I. Mironov, Strain Softening of Material in Structural Elements (UrO RAN, Ekaterinburg, 1995).

7. V. V. Struzhanov and A. V. Korkin, Herald of the USURT 3(31), 4-17 (2016).

8. V. V. Struzhanov and A. V. Korkin, Herald of the USURT 1(37), 11-19 (2018).

9. V. V. Struzhanov, A. V. Korkin, and A. E. Chaikin, J. Samara State Tech. Univ., Ser. Phys. Math. Sci. 22(4), $762-773$ (2018).

10. V. V. Struzhanov, Sib. Zh. Indust. Mat. 15(1), 138-144 (2012).

11. T. Poston and I. Stewart, Catastrophe Theory and Its Applications (Pitman, London, 1978). 